\title{
Atomic Force Microscopic Examination of Chromosomes Treated with Trypsin or Ethidium Bromide
}

\author{
Yangzhe Wu, ${ }^{a}$ Jiye CAI, ${ }^{*, a}$ Longqiu CHeng,,${ }^{a}$ Keyu Yun, ${ }^{a}$ Chenxi WAng, ${ }^{b}$ and Yong CHEN ${ }^{*, c}$ \\ ${ }^{a}$ College of Life Science and Technology, Jinan University; ${ }^{b}$ College of Science and Engineering, Jinan University; \\ Guangzhou, 510632 China: and ${ }^{c}$ Department of Microbiology and Immunology, University of Illinois; Chicago 60612, Il, \\ U.S.A. Received November 10, 2005; accepted January 5, 2006
}

Trypsin treatment is frequently used during chromosome preparation for removal of cellular contaminants, and ethidium bromide (EB) staining of bands is often used to facilitate high-resolution observations by optical microscopy. However, conventional optical microscopy is unable to visualize potential aberrations of chromosome structures caused by these physicochemical treatments. In this article, we use atomic force microscopy (AFM) in the tapping mode to obtain and analyze high-resolution images of chromosome surface structure damage associated with trypsinization and EB treatment. According to our results, the trypsin-based digestion effects became more severe as incubations increased across a range from 10 to $40 \mathrm{~s}$; a digestion time of 10 to $20 \mathrm{~s}$ appeared to be most suitable for observation by AFM. In terms of chromosomal damage induced by EB treatment, addition of EB into the media of cultured human blood cells induced chromosomal breakage in a dose-dependent fashion, and the results indicate centromeric region damnifyed severer than arms. Together, these results indicate that EB staining and the standard chromosomal preparative techniques of trypsinization can induce chromosomal damage that may affect the observed results.

Key words chromosome damage; ethidium bromide; trypsinization; atomic force microscope

Recently, researchers have sought to better understand the relationship between chromosomal structure and function. Chromosomes, primarily composed of DNA and histone proteins, must replicate prior to cell division. During replication, errors can be introduced into the DNA via physical or chemical stressors, including heat, ultraviolet radiation, and other forms of radiation, heavy metal ions, drugs and organic reagents. ${ }^{1,2)}$ However, it is also probable that chromosomal alterations might be introduced outside of replication, such as during the trypsinization or ethidium bromide (EB) staining processes ${ }^{3)}$ commonly used in visualization of chromosomes by optical microscopy. Changes of chromosome ultrastructure cannot be observed clearly under conventional optical microscopes due to technical difficulties such as diffraction limitations. However, the advent of atomic force microscopy $(\mathrm{AFM})^{4)}$ has allowed researchers to investigate small alterations in chromosome structure and morphology.

Previous studies have examined changes in chromosome structure caused by physicochemical treatments. ${ }^{5,6)}$ Acetic acid, which is frequently used to remove cellular contaminants, was applied to barley chromosomes at concentrations of 15,30 and $45 \%$. AFM analysis revealed that the optimal concentration was $30 \%$; at lower levels sufficiently clean chromosomes samples could not obtain and the chromosome surface structures could not be observed, and at higher levels the chromosome structures were significantly damaged, although the cellular contaminants were effectively removed. ${ }^{7)}$ Similar studies identified chromosomal damages induced by treatments with $\mathrm{NaCl}^{8)}$ and heavy ion radiation. ${ }^{9)}$

$\mathrm{EB}$, a heterocyclic organic compound, can insert between base pairs,${ }^{10)}$ resulting in increasing DNA rigidity and lengthening of DNA. ${ }^{11)}$ Low doses of EB can induce DNA denaturation and can lead to cancer formation. ${ }^{12)}$ Thus, it is possible that EB staining of chromosome preparations may induce structural abnormalities ${ }^{13)}$ not present in the actual organism. Up to now, though there are some works about structural changes of chromosomes caused by physicochemical treat- ments, ${ }^{3,5,6,14-16)}$ however, no previous work has used AFM to examine the effects of time-course of trypsin or different dose EB on chromosomal structures.

In this article, we used AFM in the tapping mode to examine chromosomal damage induced by EB treatment and trypsin digestion in air. Our results showed that EB could profoundly damage human chromosomes, leading to breakages and separations in a dose-dependent fashion. We also found that trypsinization could obviously change the morphological features of chromosomes with increased digestion time; the optimal digestion time for AFM analysis appeared to be $10-20 \mathrm{~s}$ under our experimental conditions, but not to be over $40 \mathrm{~s}$. In addition, our results indicate that AFM is a powerful tool for studying chromosomal damage, perhaps indicating that it will be applicable to further diagnostic applications. Together, our results indicate that common chromosomal preparative techniques, such as trypsinization and EB staining, can damage chromosomes. Thus, it is possible that some previous optical diagnoses of chromosome abnormalities may have been artifacts from the preparation process itself.

\section{Experimental}

Chromosome Preparation Cell Culture: Blood cells were obtained from women who were born without a womb or ovaries (provided by the First Subsidiary Hospital of Jinan University). Cells were cultured at $37^{\circ} \mathrm{C}$ in RPMI-1640 containing bovine serum albumin $(15 \%)$ and propagated every 3-5 d; cells undergoing logarithmic growth were used in experiments. Three samples were separated out for experimental purposes: sample A was left untreated, sample B was treated with $0.1 \mathrm{ml}$ EB (Sigma, $500 \mu \mathrm{g} / \mathrm{ml}$ ), and sample C was treated with $0.05 \mathrm{ml} \mathrm{EB}$. Cells were then incubated with or without the EB for $2 \mathrm{~h}$ prior to analysis.

Harvesting for Metaphase Chromosomes: Two drops of colchicine (Sigma, U.S.A., $20 \mu \mathrm{g} / \mathrm{ml}$, for a final concentration of $0.05 \mu \mathrm{g} / \mathrm{ml}$ ) were added to the culture $3 \mathrm{~h}$ prior to experiments, mixed gently and incubated at $37^{\circ} \mathrm{C}$. Cultures were then centrifuged at $1500 \mathrm{rpm}$ for $8 \mathrm{~min}$, and supernatants were discarded. Pellets were gently overlain with $6 \mathrm{ml}$ of $0.075 \mathrm{M}$ hypotonic $\mathrm{KCl}$ solution, incubated for $20 \mathrm{~min}$ at $37^{\circ} \mathrm{C}$, and centrifuged again at $1500 \mathrm{rpm}$ for $8 \mathrm{~min}$. The supernatant was discarded, the pellet was resuspended in $2 \mathrm{ml}$ of freshly prepared fixative ( $3: 1$ methanol : acetic acid), and 

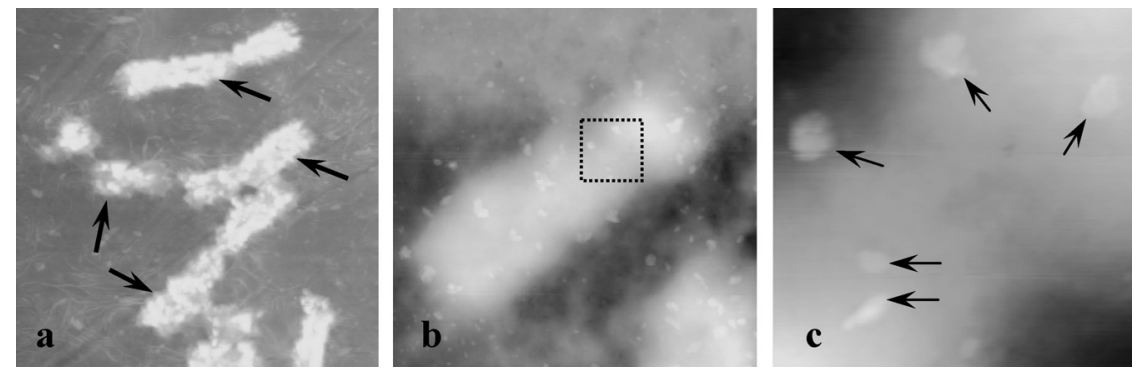

Fig. 1. Chromosomal AFM Images without Trypsin Treatment

Chromosomal ultrastructures concealed by cellular contaminants and debris or protein particles (black arrows). Figure $\mathrm{c}$ is enlarged view according to broken square frame in Fig. b. Scan ranges (a) $15 \mu \mathrm{m}$, (b) $5 \mu \mathrm{m}$, (c) $1 \mu \mathrm{m}$.
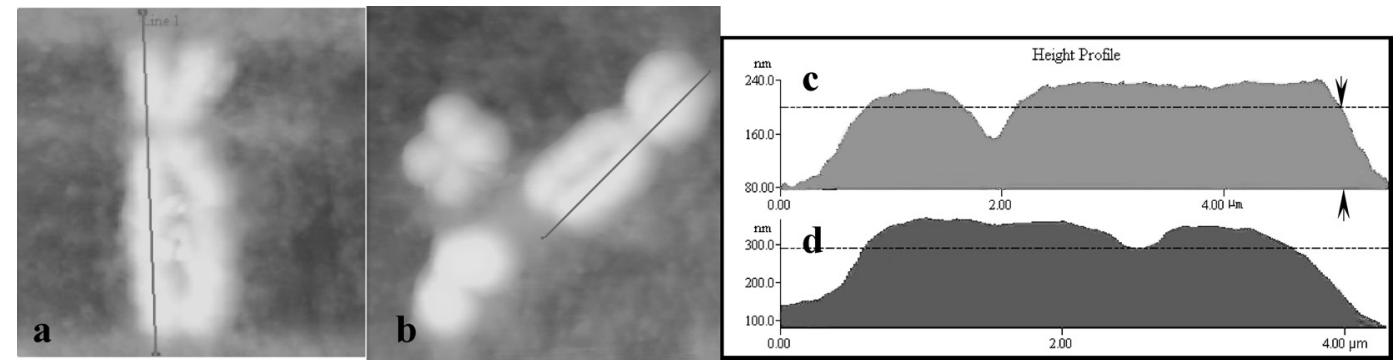

Fig. 2. AFM Images and Height Profile of Chromosomes Digested by Trypsin for $10 \mathrm{~s}$

The height profile (c, d) were generated along the lines shown in Figs. a, b, and the average height (difference between the dark broken lines and the bottom in Fig. $c$ ) along the lines is about $160 \mathrm{~nm}$ (a) and $270 \mathrm{~nm}$ (b), respectively. Scan ranges (a) $5.5 \mu \mathrm{m}$, (b) $6 \mu \mathrm{m}$.
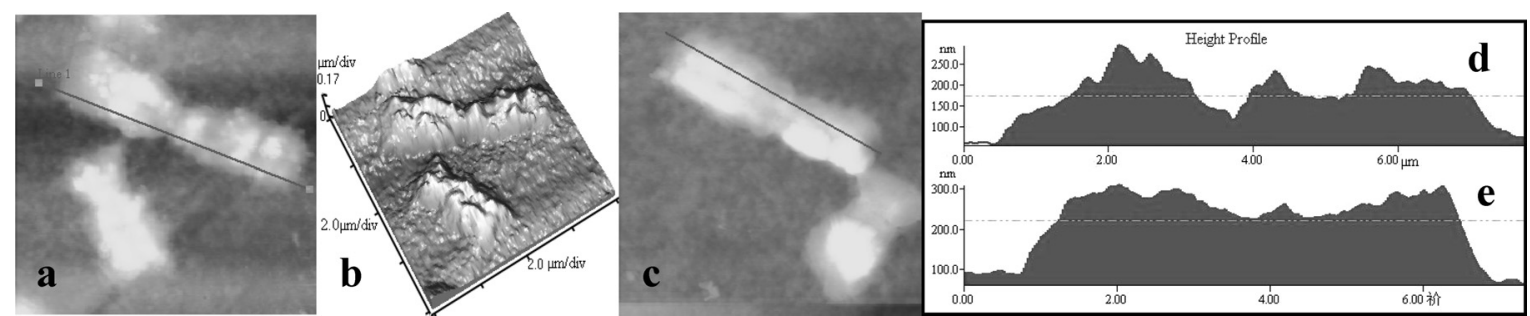

Fig. 3. Morphological and Three-Dimensional Chromosomal Images Digested by Trypsin for $20 \mathrm{~s}$

The height profile (d, e) were generated along the line shown in Figs. a, c, and the average height along the lines is about $120 \mathrm{~nm}$ (a) and $155 \mathrm{~nm}$ (c), respectively. Scan range: (a,b) $8 \mu \mathrm{m}$

the sample was centrifuged at $1500 \mathrm{rpm}$ for $8 \mathrm{~min}$. This step was repeated twice, with the second step using $5 \mathrm{ml}$ of freshly prepared fixative. Pellets were then resuspended in $0.5 \mathrm{ml}$ of fixative and $3-4$ drops of this cell suspension were dropped to glass slides from a height of $30-50 \mathrm{~cm}$ (for even spreading). Slides were air dried, baked in a drying oven for $2 \mathrm{~h}$ at $70^{\circ} \mathrm{C}$, then immersed in Earl's solution ( $\left.\mathrm{pH}=6.20,87.5^{\circ} \mathrm{C}, 0.25 \%\right)$. Four "A" samples were immersed in trypsin $(0.1 \% ; \mathrm{pH}=7.0)$ for $10,20,30$ and $40 \mathrm{~s}$, respectively. "B" and "C" sample were immersed in trypsin for 10 and $20 \mathrm{~s}$ All slides were then stained with Giemsa (10\%) for $8 \mathrm{~min}$ and air-dried.

AFM Imaging The prepared samples were imaged at room temperature using an AF microscope (CP-Research, U.S.A.) in tapping mode in air. The curvature radius of the silicon nitride tip was about $10 \mathrm{~nm}$, the force constant was about $2.8 \mathrm{~N} / \mathrm{m}$, the scanning range was $100 \mu \mathrm{m}$, and all of the images were flatted with the provided software to eliminate low-frequency background noise during scanning direction.

\section{Results and Discussion}

Chromosomes without Trypsin Treatment To investigate the effect of trypsin treatment on chromosomal structure, a control experiment was performed (Fig. 1). The results indicate that trypsin treatment is indispensable during chromosome preparation for removal of cellular contaminant or debris, because those contaminant or debris could conceal the chromosomal ultrastructures (Fig. 1, white particles, black arrows).

Time-Course Observations of Trypsin Digestion of Chromosomal Spreads Then, the effects of trypsin treatment on chromosome surface structures were investigated by AFM. And AFM images of chromosomes digested by trypsin for 10, 20, 30 and $40 \mathrm{~s}$ are shown in Figs. 2-5, respectively. Spreads digested for $10 \mathrm{~s}$ contained chromosomes that were shortened in both length and breadth. As the digestion time increased, we observed trypsin-induced changes in chromosome morphology and surface ultrastructure. Digestion for 10 and $20 \mathrm{~s}$ induced very little damage (Figs. 2, 3). After $30 \mathrm{~s}$ digestion, the morphological changes are more apparent, chromosomal figure obscured (Figs. 4a, b), one arm almost completely digested by trypsin (Fig. 4a; white arrow), and chromosomal gap induced by trypsin digestion can be observed (Fig. 4a; small black arrow). And when the digestion duration increased and achieved $40 \mathrm{~s}$, the morphological structure of chromosome changed dramatically (Fig. 5). The trypsin-based digestion effects became more severe as incubations increased across a range from 10 to $40 \mathrm{~s}$, then the av- 

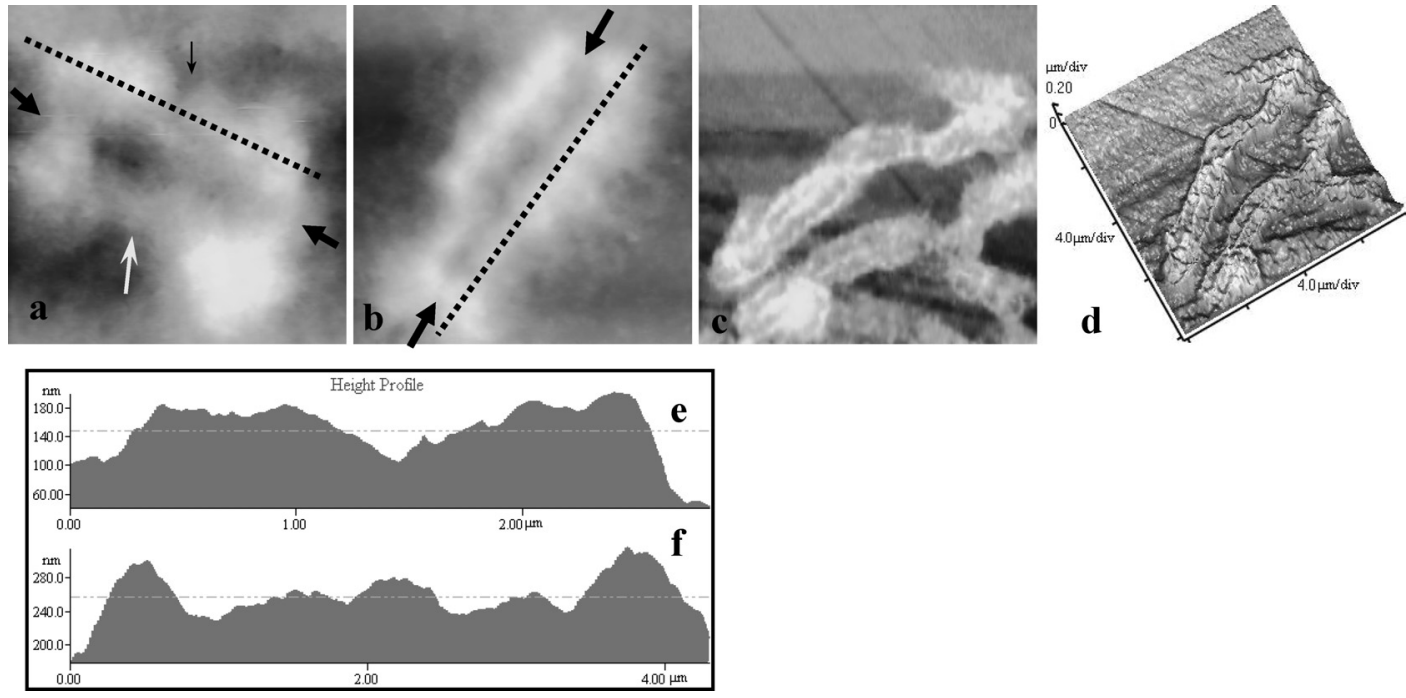

Fig. 4. Morphological and Three-Dimensional Chromosomal Images Digested by Trypsin for $30 \mathrm{~s}$

The height profile (e, f) were generated along the line shown in Figs. a, b, and the average height along the lines is about $120 \mathrm{~nm}$ (a) and $115 \mathrm{~nm}$ (b), respectively. Figure d is outline drawing of Fig. c showing the 'ghost strands.' Scan ranges (a) $3 \mu \mathrm{m}$, (b) $4 \mu \mathrm{m}$, (c) $15 \mu \mathrm{m}$.
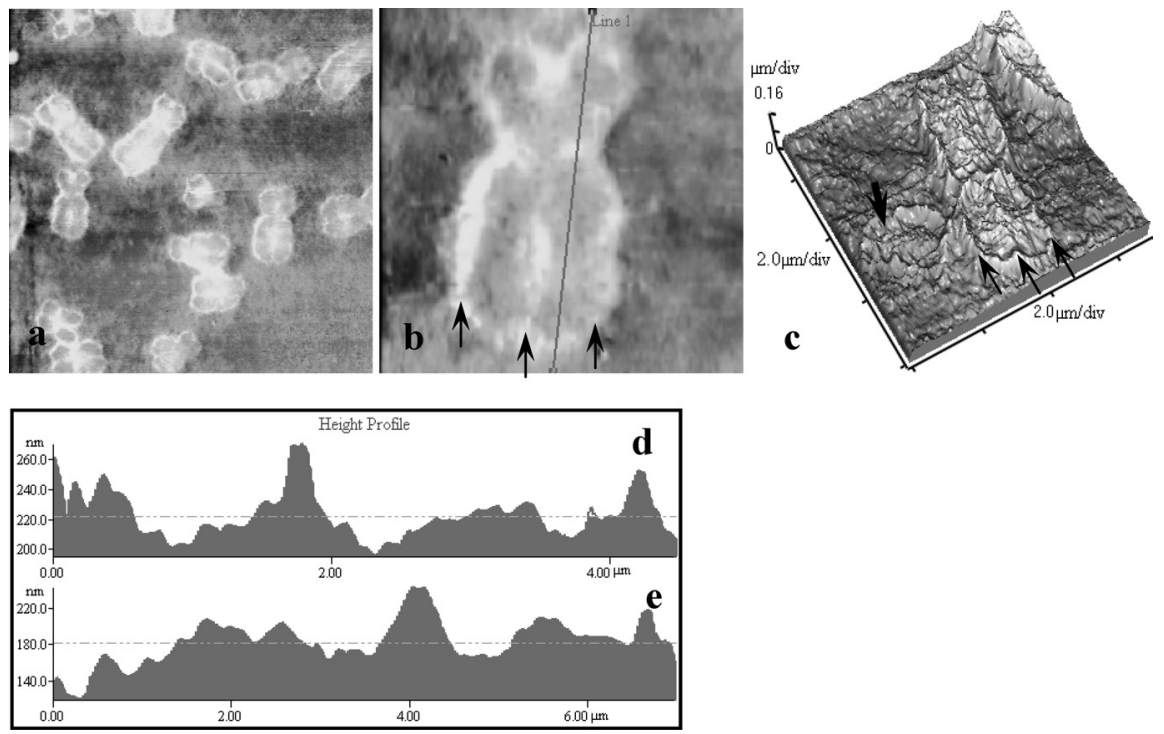

Fig. 5. Morphological (a, b) and Three-Dimensional (c) Chromosomal Images Digested by Trypsin for $40 \mathrm{~s}$

The height profile (d, e) were generated along the line shown in Figs. b, c, and the average height along the lines is about $25 \mathrm{~nm}$ (b) and $50 \mathrm{~nm}$ (c), respectively. And the "ghost strands" are shown in the Figs. b, c (small black arrows), remains of chromosome indicated by big black arrow in Fig. c. Scan ranges (a) $20 \mu \mathrm{m}$, (b) $4.5 \mu \mathrm{m}$, (c) $7 \mu \mathrm{m}$.

erage height which is the difference between the dark broken lines and the bottom in Height Profiles were analyzed. By our statistical results according to large numbers of AFM images, the average height of chromosomes decreased from about $180 \mathrm{~nm}(10 \mathrm{~s}), 136 \mathrm{~nm}(20 \mathrm{~s})$, and $109 \mathrm{~nm}(30 \mathrm{~s})$ to about $50 \mathrm{~nm}(40 \mathrm{~s})$. As all other conditions were held consistent, our results suggest that these changes are in response to trypsinization. This is logical, as trypsinization digests the histones that largely define the morphology and volume of the chromosome. As our results indicate that the damage increases with trypsinization time, it is valuable to assess optimal digestion times for AFM scanning. Our observations indicated that 10 to $20 \mathrm{~s}$ trypsin digestion is optimal for AFM scanning. At this time, the protein residue can be easily wiped off the slide, but the chromosomal damage appears minimal.
Interestingly, our AFM examinations revealed some unique chromosomal morphology. In particular, we observed what appeared to be three chromosomal strands in some frames (Fig. 4c, with emphasis provided in 4d). As triploidy is a lethal chromosomal aberration, the most likely explanation for this observation is that these are 'ghost strands,' which are the remains mainly composed of DNA and the DNA/histone complex after trypsinization. This hypothesis is supported by the results of the longer ( $40 \mathrm{~s}$ ) digestion (Fig. 5), wherein the two chromosome arms darkened and decreased very dramatically. The ghost strands (indicated by black arrows in Figs. 5b, c) are likely to be the remains composed of DNA and its enwinding conjugation with histone. In addition, double-centromere chromosomes, which necessarily related with chromosomal aberrations, were also observed (Figs. 4a, b, big black arrows). 

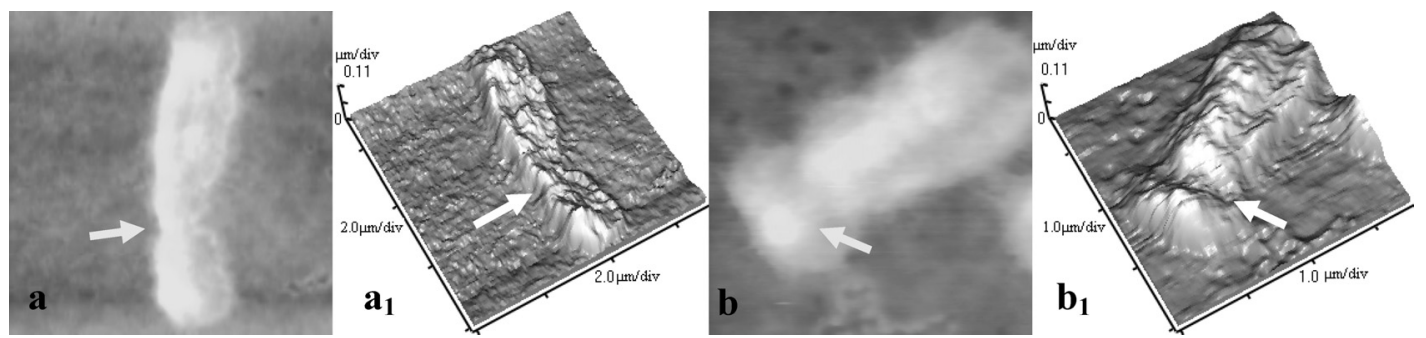

Fig. 6. Morphological and Three-Dimensional Chromosomal Images Treated with $0.05 \mathrm{ml}$ EB and Then Digested by Trypsin for $20 \mathrm{~s}$ Big white arrows indicate the centromeric region. Scan ranges (a) $7 \mu \mathrm{m}$, (b) $6 \mu \mathrm{m}$.
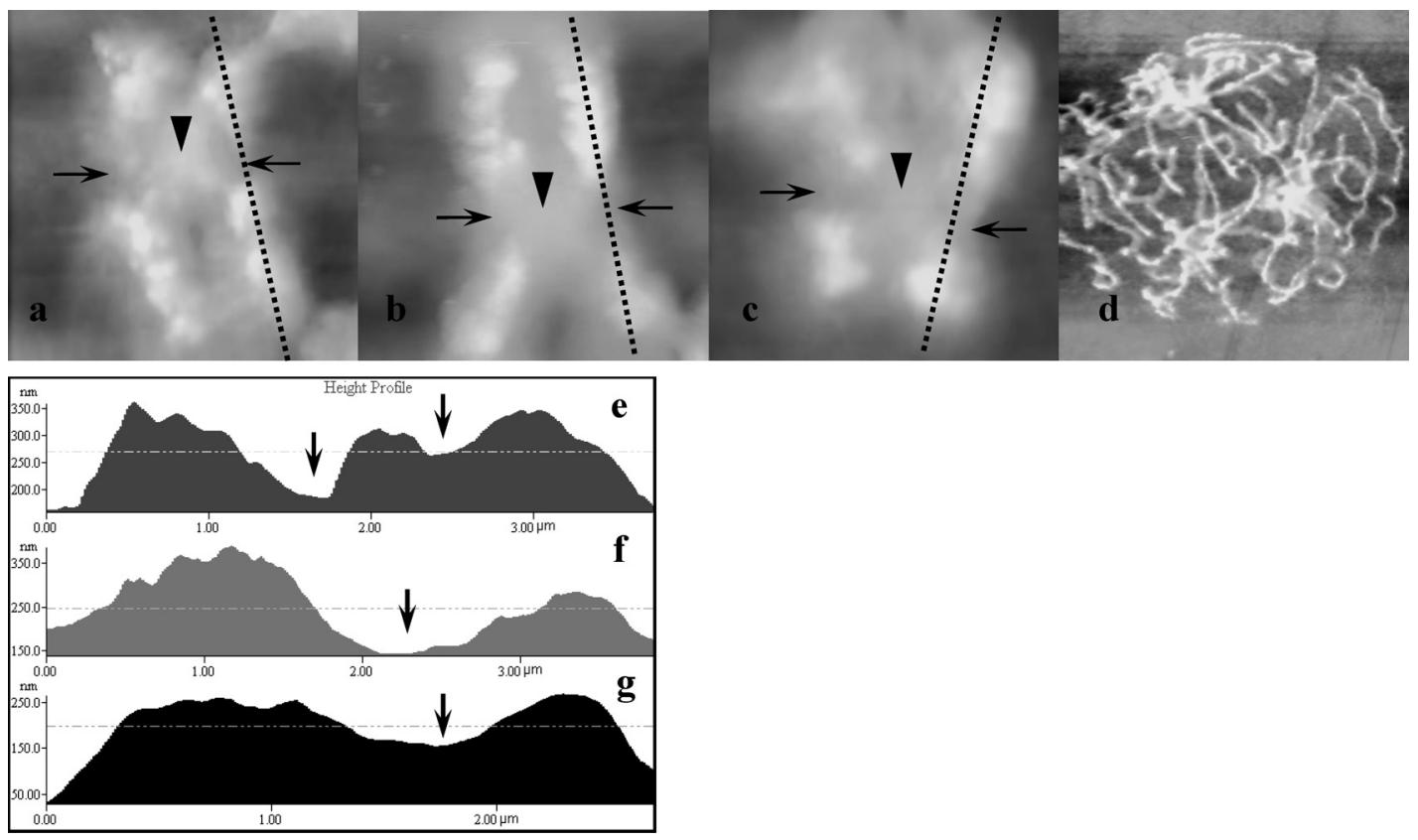

Fig. 7. Topographic and Three-Dimensional Chromosomal Images Treated with $0.1 \mathrm{ml} \mathrm{EB}$ and Digested by Trypsin for $20 \mathrm{~s}$

The height profile $(\mathrm{e}-\mathrm{g})$ were generated along the line shown in Figs. a, b, c, respectively. Arrows indicate chromosomal damage, where the arms have been broken or divided from the centromere (big black arrows). The EB treated chromatins were unable to achieve metaphase shown in Fig. d, the average width and height of chromatins were about $660 \mathrm{~nm}, 115 \mathrm{~nm}$, respectively. Arrows in height profile indicate the damage location of chromosomal arms. Scan ranges (a,b) $4 \mu \mathrm{m}$, (c) $3 \mu \mathrm{m}$, (d) $25 \mu \mathrm{m}$.

And finally, from the discussion above, we can conclude that (1) trypsin can change the chromosomal surface structure with the digestion duration increased, however, chromosomal cleavage and breakage did not happen during this control experiment; (2) 10 to $20 \mathrm{~s}$ trypsin digestion is optimal for AFM scanning.

Effects of Different Dose EB on Chromosomes To examine the effects of EB staining on chromosome ultrastructure, we used AFM to examine chromosomes treated with various volumes $(0.05,0.1 \mathrm{ml})$ of $\mathrm{EB}$ for $2 \mathrm{~h}$ and then digested with trypsin for $20 \mathrm{~s}$. The observed chromosomal damage caused by $0.05 \mathrm{ml}$ EB (Fig. 6) was less severe than that caused by $0.1 \mathrm{ml}$ (Fig. 7), indicating that the chromosomal damage induced by EB is dose-dependent under our experimental conditions, and $0.1 \mathrm{ml} \mathrm{EB}$ treatment could induce the chromosomal cleavage from centromere (Figs. $7 \mathrm{a}-\mathrm{c}$, big black arrowheads) and rupture of chromosomal arms (Fig. 7, small black arrows).

In addition, we observed morphological differences between chromosomes in groups " $\mathrm{A}$ ", "B" and " $\mathrm{C}$ ", which corresponded to different action. "A" was just digested by trypsin (control experiment), and "B" and "C" acted with EB and then digested by trypsin. During cell differentiation, chromosomes undergo cyclic changes such as condensation and extension. Condensation is highest during metaphase; it is during this time that the topographical structures of the chromosomes can be clearly visualized by AFM. However, many of the EB treated chromosomes (actual chromatins) were unable to achieve metaphase, but were still observable by AFM (Fig. 7d), and its average width and mean height were about 660 and $115 \mathrm{~nm}$ respectively by measuring. This indicates that prophase chromosomes can be analyzed by AFM, suggesting a new application of this technique to molecular biology.

Comparison of chromosome morphologies in samples without (Figs. 2-5) and with (Figs. 6, 7) EB treatment revealed that $E B$ could interdict cell differentiation by interfering with chromosome condensation. And virtually, EB is a cationic dye that can inhibit DNA synthesis by intercalating into the base pairs of DNA ${ }^{17-19)}$ and change its physical properties such as rigidity, elasticity, space structure etc., ${ }^{10,11,20-22)}$ and even result in DNA molecules broken (this is approved by our other experiment about DNA damage induced by EB), then chromosomal structure damnifyed. The results indicate chromosome damage mainly locate at centromeric region (Fig. 7), which is likely correlated with the 
higher density of DNA. ${ }^{23)}$ The region contains higher density of DNA molecules, which are intercalated by more EB molecules, will be damnifyed severer than that containing lower density of DNA.

Our results indicate AFM, a "nanodetector," is an appropriate tool to study the damnification and structural denaturation of gene materials such as chromosome and DNA, and probably possess the potential application in gene disease diagnosing.

In samples pre-treated with $\mathrm{EB}$ and then digested by trypsin, we observed increased chromosomal damage, an effect that is known as 'double acting,' which made the damage more severe than one factor such as only trypsin or EB. It indicated that less treatment process in chromosome preparation process is optimal for AFM imaging, such as Giemsastaining is unnecessary ${ }^{9)}$ and it can conceal chromosomal ultrastructure.

\section{Conclusions}

In this paper, we used AFM to study the effects of trypsinization on chromosome morphology and structure. Our results revealed that the effects of digestion on the chromosomes increased in a time-dependent fashion. For AFM analysis, digestion with trypsin for 10 to $20 \mathrm{~s}$ was found to be optimal, yielding clean, relatively undamaged chromosomes. When the digestion duration increased and achieved $40 \mathrm{~s} \mathrm{(ac-}$ cording to large numbers of AFM images), the damage increased to such a point that the chromosomes were no longer suitable for AFM imaging.

We also studied chromosomal damage and abnormalities in metaphase spreads induced by EB treatment, which is a commonly used molecular biological technique that may damage chromosomes and/or inhibit cell differentiation. AFM analysis revealed that EB treatment could induce chromosomal breakage and cleavage (Fig. 7), providing important proof for the bio-toxicity of EB. ${ }^{24-26)}$ The results indicate chromosome damage mainly locate at centromeric region and centromeric region damnifyed severer than arms (Fig. 7), which is likely correlated with the higher density of DNA. ${ }^{19)}$ The region contains higher density of DNA molecules, which are intercalated by more EB molecules, will be damnifyed severer than that containing lower density of DNA.

Overall, our results revealed that AFM is capable of visualizing various chromosomal damages that are not generally discriminated by conventional optical microscopy. Combination of AFM analysis and appropriate chemical treatments will likely provide important insights into the organization of higher-order chromosomal structures and the aberrations induced by chemical treatments. ${ }^{27)}$ In this way, AFM examination of unstained chromosomal samples may provide us with new understandings of the structure/function relationships in chromosomes. ${ }^{28)}$ Furthermore, AFM and other new technolo- gies such as scanning near-field optical microscopy ${ }^{19,29,30)}$ may allow more in-depth examinations of karyotypes and/or associations between chromosomal aberrations and disease in the future.

Acknowledgement This research project is supported by the national natural science foundation of China (No.60278014, 30230350) and the natural science foundation of Guangdong province (No. 36704).

\section{References}

1) Akao T., Fukumoto T., Ihara H., Ito A., FEBS Lett., 391, 215-218 (1996).

2) Zimmer C., Luck G., Triebel H., Biopolymers, 13, 425-453 (1974).

3) Ronne M., J. Dairy Sci., 72, 1363-1377 (1989).

4) Binning G., Quate C. F., Gerver C., Phys. Rev. Lett., 56, 930-933 (1986).

5) Fritzsche W., Microsc. Res. Techniq., 44, 357-362 (1998).

6) Hoshi O., Owen R., Miles M., Ushiki T., Cytogenet. Genome. Res., 107, 28-31 (2004).

7) Sugiyama S., Yoshino T., Kanahara H., Shidhiri M., Fukushi D., Otani T., Anal. Biochem., 324, 39-44 (2004).

8) Liu X. Q., Sugiyamaa S., Xu Q. Y., Kobori T., Hagiwara S., Ohtani T., Ultramicroscopy, 94, 217-223 (2003).

9) Murakami M., Minamihisamatsu M., Sato K., Hayata I., J. Biochem. Biophys. Method, 48, 293-301 (2001).

10) Geall A. J., Blagbrough I. S., J. Pharmaceut. Biomed., 22, 849-859 (2000).

11) Manning G. S., Quart. Rev. Biophys., 11, 179-246 (1978).

12) Gupta R. S., Gupta R., Goldstein S., Exp. Gerontol., 31, 267-280 (1996).

13) Ikeuchi T., Cytogenet. Cell Genet., 38, 56-61 (1984).

14) Darzynkiewicz Z., Kapuscinski J., Cytometry, 9, 7-18 (1988).

15) De Groogh B. G., Putman C. A., J. Microsc., 168, 239-247 (1992).

16) Fritzsche W., Augustin R., Michel S., Claussen U., J. Computer-Assisted Microsc., 10, 71-76 (2000).

17) Tuteja N., Phan T. N., Biochem. Biophys. Res. Commun., 244, 861867 (1998).

18) Nordmeier E., J. Phys. Chem., 96, 6045-6055 (1992).

19) Lybrand T., Kololman P., Biopolymers, 24, 1863-1879 (1985).

20) Pope L. H., Davies M. C., Laughton C. A., Roberts C. J., Tendler S. J. B., Williams P. M., Anal. Chim. Acta, 400, 27-32 (1999).

21) Pope L. H., Davies M. C., Laughton C. A., Roberts C. J., Tendler S. J. B., Williams P. M., J. Microsc., 199, 68-78 (2000).

22) Utsuno K., Tsuboi M., Katsumata S., Iwamoto T., Chem. Pharm. Bull., 49, 413-417 (2001).

23) Yoshino T., Sugiyama S., Hagiwara S., Fukushi D., Shichiri M., Nakao H., Kim J. M., Hirose T., Muramatsu H., Ohtani T., Ultramicroscopy, 97, 81-87 (2003).

24) Armand R., Channon J. Y., Kintner J., White K. A., Miselis K. A. Perez R. P., Lewis L. D., Toxicol. Appl. Pharmacol., 196, 68-79 (2004).

25) Guo J., Sun Y. J., Huazhong Univ. Sci. Technolog. Med. Sci., 22, 180$182(2002)$

26) Ohta T., Tokishita S., Yamagata H., Mutat. Res., 492, 91—97 (2001)

27) Sun Y. C., Arakawa H., Osada T., Ikai A., Appl. Suf. Sci., 188, 499505 (2002)

28) Fritzsche W., Takac L., Henderson E., Crit. Rev. Eukar. Gene, 7, 231240 (1997)

29) Kirsch A. K., Subramaniam V., Striker G., Schnetter C., Arndt-Jovin D. J., Jovin T. M., Biophys. J., 75, 1513-1521 (1998).

30) Hausmann M., Liebe B., Perner B., Jerratsch M., Greulich K. O., Scherthan H., Micron, 34, 441-447 (2003). 